DANILLO SILVA MARCON

Farmacovigilância veterinária baseada em relatos espontâneos de uma empresa farmacêutica no Brasil

São Paulo, 2019 


\section{Farmacovigilância veterinária baseada em relatos espontâneos de uma empresa farmacêutica no Brasil}

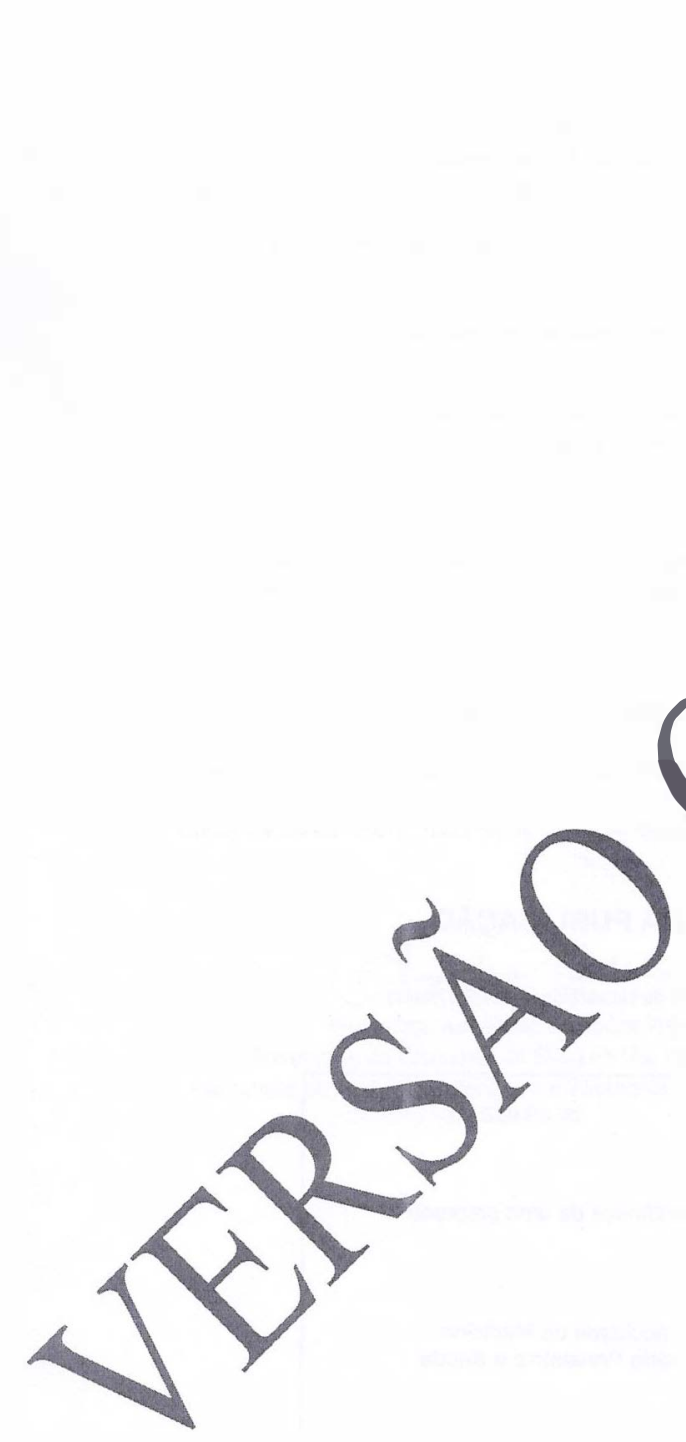

Dissertação apresentada ao piograıná de Pós-Graduação ex Epic mologia Experimental Aplicač às Zoonoses da Faculdade de Meodicinta Veterinária e Zootecnia da Yn versıo deje São Paulo para obtençádo "ulo oe Mestre

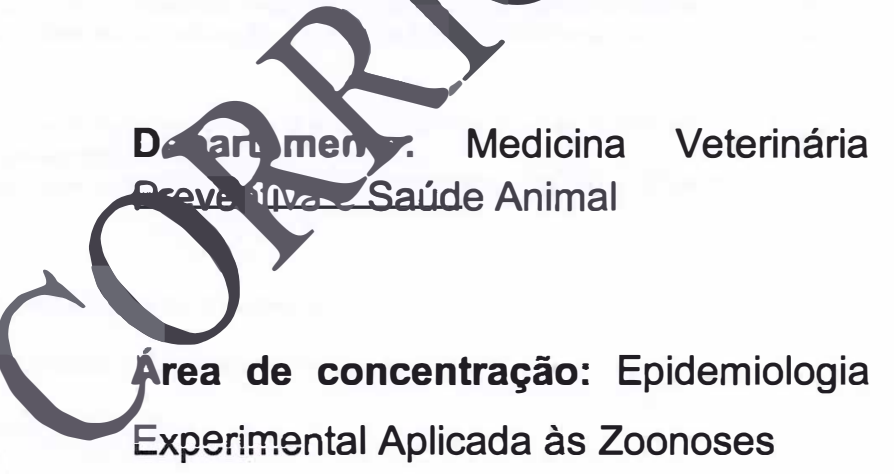

Orientador: Prof. Dr. Oswaldo Santos Baquero

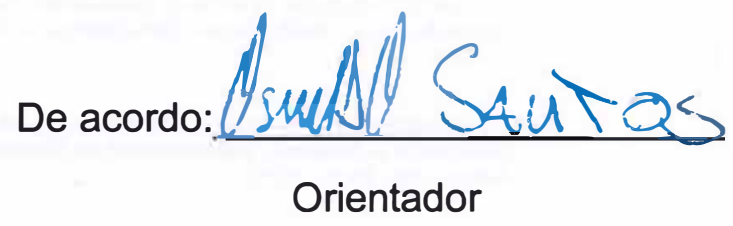

São Paulo, 2019

Obs: A versão original encontra-se disponível na Biblioteca da FMVZJUSP 
Autorizo a reprodução parcial ou total desta obra, para fins acadêmicos, desde que citada a fonte.

DADOS INTERNACIONAIS DE CATALOGAÇÃO NA PUBLICAÇÃO

(Biblioteca Virginie Buff D’Ápice da Faculdade de Medicina Veterinária e Zootecnia da Universidade de São Paulo)

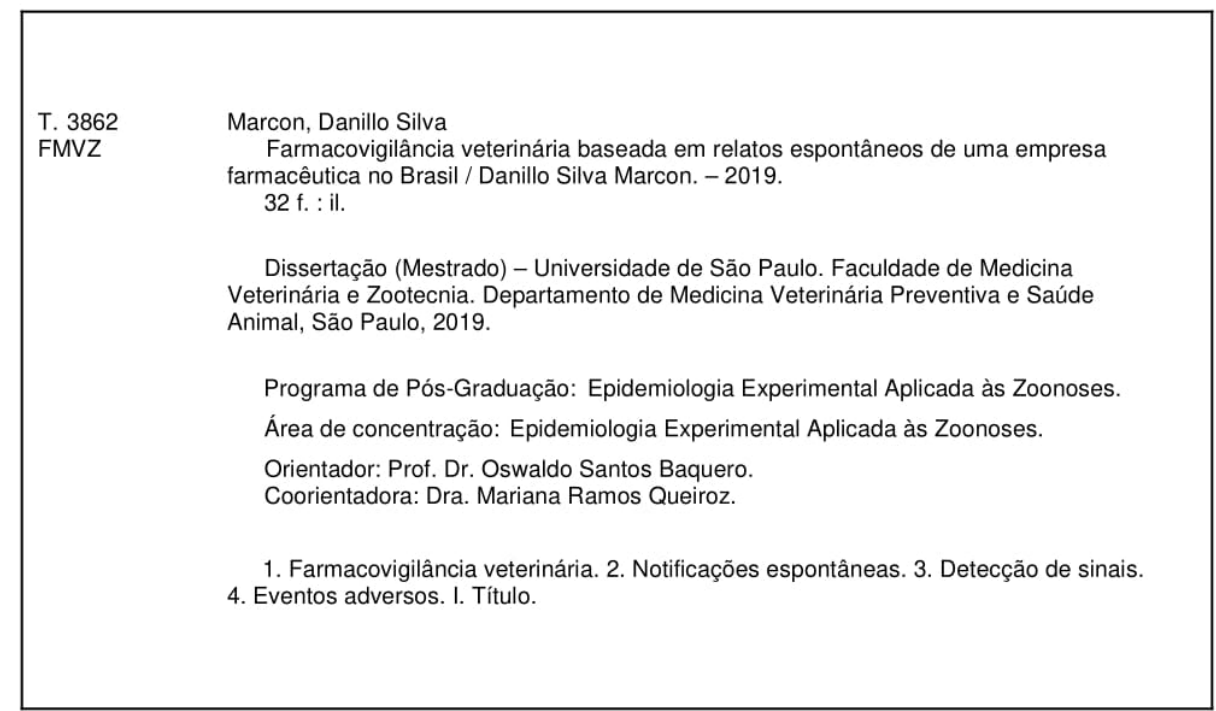

Ficha catalográfica elaborada pela bibliotecária Sandra Regina Toledo, CRB-8/8146, da FMVZ/USP. 


\section{Certificado da Comissão de Ética}

\section{UNIVERSIDADE DE SÃo PAULO}

Comissão de Ética no Uso de Animais

São Paulo, 26 de setembro de 2019

CEUAx N 8238150318

IImo(a). Sr(a).

Responsável: Oswaldo Santos Baquero

Área: Medicina Veterinária Preventiva E Saúde Animal

Oswaldo Santos Baquero (orientador)

Título do projeto: "Farmacovigilância veterinária baseada em relatos espontâneos de uma empresa farmacêutica no Brasil".

Parecer Consubstanciado da Comissão de Ética no Uso de Animais FMVZ $(D 000797)$

A Comissão de Ética no Uso de Animais da Faculdade de Medicina Veterinária e Zootecnia da Universidade de São Paulo, no cumprimento das suas atribuições, ANALISOU e APROVOU a Alteração do cadastro (versão de 02/setembro/2019) do protocolo de estudo acima referenciado.

Resumo apresentado pelo pesquisador: "Há um erro de digitação no título. O termo "relatórios" deve ser substituído por "relatos" e no título em inglês deve haver um hífen entre "spontaneous" e "reports".

Aproveitei para alterar "companhia" por "empresa" pois o último termo caracteriza melhor a fonte dos dados utilizados.

Os títulos atualizados devem ser:

Farmacovigilância veterinária baseada em relatos espontâneos de uma empresa farmacêutica no Brasil

Spontaneous-reports-based veterinary pharmacovigilance of a pharmaceutical company in Brazil".

Comentários da CEUA: "trata-se de correção de digitação no título.".

$$
\text { Mnneliese Tealar. }
$$

Profa. Dra. Anneliese de Souza Traldi Presidente da Comissão de Ética no Uso de Animais

Faculdade de Medicina Veterinária e Zootecnia da Universidade de São Paulo

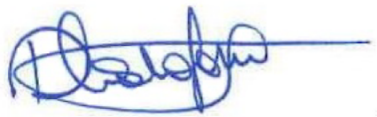

Roseli da Costa Gomes Secretária

Faculdade de Medicina Veterinária e Zootecnia da Universidade de São Paulo 


\section{FOLHA DE AVALIAÇÃO}

Autor: MARCON, Danillo Silva

Título: Farmacovigilância veterinária baseada em relatos espontâneos de uma empresa farmacêutica no Brasil

Dissertação apresentada ao programa de Pós-Graduação em Epidemiologia Experimental Aplicada às Zoonoses da Faculdade de Medicina Veterinária e Zootecnia da Universidade de São Paulo para obtenção do título de Mestre

data

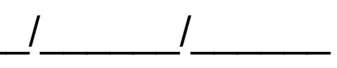

Banca Examinadora

Prof. Dr.

Instituição:

Julgamento:

Prof. Dr.

Instituição:

Julgamento:

Prof. Dr.

Instituição:

Julgamento: 


\section{DEDICATÓRIA}

Dedico este trabalho aos meus pais Celso e Nilza e a toda minha família que, com muito carinho e apoio, não mediram esforços para que eu chegasse até esta etapa da minha vida. Dedico também à minha esposa, Luisa, que de forma especial e carinhosa me deu força e coragem, me apoiando nos momentos de dificuldades, possibilitando a realização de um sonho. 


\section{AGRADECIMENTOS}

Agradeço aos docentes da Universidade de São Paulo, principalmente da faculdade de Medicina Veterinária e Zootecnia, que auxiliaram no desenvolvimento deste trabalho que traz à tona o conceito "Farmacovigilância", perpetuando a evolução da medicina veterinária.

Agradeço ao meu orientador, Prof. Dr Oswaldo pela insistência e apoio desde o início do desenvolvimento do tema.

Agradeço à minha coorientadora, Dra. Mariana que trouxe mais compreensão nas conversas frente às adversidades pessoais que passei.

Agradeço aos meus amigos, pelas alegrias, tristezas e dores compartilhadas. Com vocês, as pausas entre um parágrafo e outro de produção melhora tudo o que tenho produzido na vida. 


\section{RESUMO}

MARCON, D.S. Farmacovigilância veterinária baseada em relatos espontâneos de uma empresa farmacêutica no Brasil. 2019. 32 f. Dissertação (Mestrado em Ciências) - Faculdade de Medicina Veterinária e Zootecnia, Universidade de São Paulo, São Paulo, 2019.

A farmacovigilância de uma empresa veterinária pode compreender a análise de relatos espontâneos de eventos adversos (EA) relacionados aos seus produtos. No presente estudo foi elaborado um fluxograma de classificação de EA que foi usado para analisar EA notificados ao serviço de atendimento ao cliente e farmacovigilância de uma empresa veterinária no Brasil. Os binômios produto-EA foram caracterizados em termos das suas frequências e posteriormente foram utilizados três modelos de detecção de sinais: Reporting Odds Ratio, Bayesian confidence propagation neural network, e Gamma Poisson Shrinker. Os sinais detectados com os três métodos foram classificados de acordo com a sua intensidade, sempre com o sinal mais intenso na primeira posição. Entre os sinais detectados pelos três métodos, as posições de cada sinal foram somadas para obter uma classificação agregada que levasse em consideração os resultados dos três métodos e permitisse uma interpretação serial. Entre os 531 relatos foram identificados 20 EA, 54 binômios produto-EA e 34 binômios produto-reação adversa medicamentosa. Do total de relatos 7 foram sinais identificados pelos três métodos utilizados. A classificação de EA seguindo critérios explícitos e o uso combinado de mais de um método de detecção de sinais aprimoram a farmacovigilância baseada em relatos espontâneos.

PALAVRAS-CHAVE: FARMACOVIGILÂNCIA VETERINÁRIA. NOTIFICAÇÕES ESPONTÂNEAS. DETECÇÃO DE SINAIS. EVENTOS ADVERSOS 


\begin{abstract}
MARCON, D.S. Spontaneous-reports-based veterinary pharmacovigilance of a pharmaceutical company in Brazil. 2019. 32 P. Dissertation (Master of Science) University of Veterinary Medicine and Animal Science, University of São Paulo, São Paulo, 2019.
\end{abstract}

The pharmacovigilance of a veterinary company may include the analysis of spontaneous reports of adverse events $(A E)$ related to its products. The present study developed an AE classification flowchart to analyze AE notified to the customer service and pharmacovigilance of a veterinary company in Brazil. The product-AE binomials were characterized in terms of their frequencies and subsequently, three signal detection models were used: Reporting Odds Ratio, Bayesian confidence propagation neural network, and Gamma Poisson Shrinker. The signals detected with the three methods were classified according to their intensity, always with the most intense signal in the first position. Among the signals detected by the three methods, the positions of each signal were summed to obtain an aggregated classification that took into account the results of the three methods and allowed a serial interpretation. Among the 531 reports, 20 AE, 54 product-AE binomials and 34 product-Adverse Drug Reaction binomials were identified. From the total of reports, seven were signs identified by the three methods. The classification of AE following explicit criteria and the combined use of more than one signal detection method enhances spontaneous-reports-based pharmacovigilance.

KEYWORDS: VETERINARY PHARMACOVIGILANCE. SPONTANEOUS NOTIFICATIONS. SIGNAL DETECTION. ADVERSE EVENTS 


\section{LISTA DE FIGURAS}

Figura $1 \quad$ - $\quad$ Fluxograma para classificar eventos adversos.................15 


\section{LISTA DE TABELAS}

Tabela 1 - Frequência dos relatos do binômio produto-EA. São Paulo, 2019.

Tabela 2 - Frequência dos relatos do binômio produto-RAM. São Paulo, 2019.

Tabela 3 - Frequência dos relatos do binômio produto e reações adversas a medicamentos inesperadas. São Paulo, 2019.

Tabela 4 - Frequência dos erros de medicação notificados. São Paulo, 2019.

Tabela 5 - Classificação dos sinais detectados de acordo com a força do sinal. São Paulo, 2019 


\section{SUMÁRIO}

1 INTRODUÇÃO

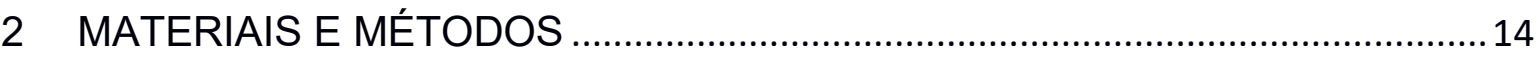

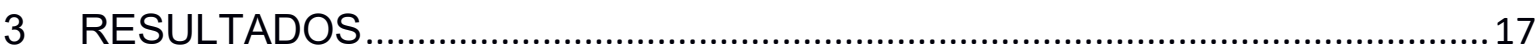

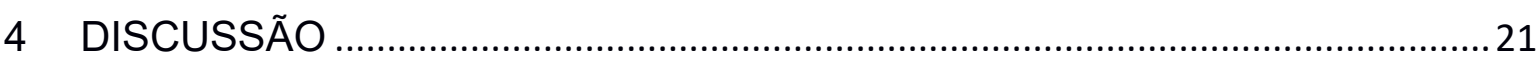

5 CONCLUSÃO

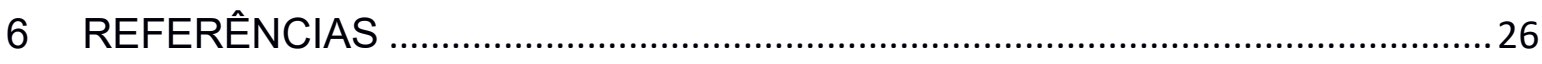

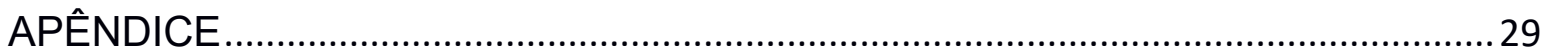




\section{INTRODUÇÃO}

Quando um produto farmacêutico é autorizado pela primeira vez para uso na prática clínica, as informações referentes ao seu uso geralmente são obtidas em ensaios clínicos que apoiam o pedido de autorização de introdução no mercado (EMA, 2016). Embora esses estudos sugiram uma relação benefício/risco satisfatória sob condições controladas, alguns eventos adversos (EA) acabam sendo detectados só após a autorização e comercialização. Isso cria a necessidade de sistemas de farmacovigilância que abranjam a fase pós-autorização.

Segundo a WHO (2018), farmacovigilância é a ciência relativa à detecção, avaliação, compreensão e prevenção de eventos adversos relacionados aos produtos. Os EA incluem qualquer sintoma ou anormalidade laboratorial decorrente de reações adversas a medicamentos (RAM) (LIMA et al, 2010, EMA, 2017), inefetividade ou falha terapêutica, intoxicações medicamentosas, erros de medicação, interação medicamentosa, uso abusivo de produtos ou uso não aprovado de produtos pela autoridade sanitária (MINISTÉRIO DA SAÚDE, 2014b; ANVISA 2009, MOTA, 2017). Os relatos espontâneos (notificações recebidas pelas empresas farmacêuticas ou órgãos regulatórios) são uma fonte de informação dos possíveis EA causados pelos produtos comercializados. São amplamente usados na farmacovigilância e junto com outras fontes de informação, como a literatura científica, sites públicos, redes sociais e outros, são a base da detecção de sinais, isto é, de associações potencialmente causais entre o uso de produtos comercializados e EA (CIOMS, 2010).

Como forma de instituir um programa de farmacovigilância no mercado veterinário brasileiro, o Ministério da Agricultura, Pecuária e Abastecimento (MAPA) pôs à consulta pública a portaria SDA 152/08, onde apresentou a normativa de um sistema de farmacovigilância veterinária nacional. A portaria SDA 152/08 caracterizava o MAPA como órgão responsável por receber e analisar os EA para fornecer informação ao usuário, além de elaborar cenários e verificar tendências relacionadas aos EA identificados. Seria sua função responder aos relatos recebidos e determinar as medidas preventivas e corretivas que deveriam ser realizadas pelo estabelecimento fabricante (FUSCO, OLIVEIRA, PEPE, 2010). A normativa não prevaleceu e sua publicação foi postergada, comprometendo tais avaliações e fiscalizações. Como resultado, os padrões de ocorrência dos EA 
continuam sendo desconhecidos e a demanda por ações de mitigação persistem. As empresas farmacêuticas podem implementar seus próprios sistemas de farmacovigilância e com isso facilitar a criação de um futuro sistema nacional de farmacovigilância.

O presente trabalho teve como objetivo implementar um procedimento de classificação de EA com posterior detecção de sinais por meio de 3 métodos cujos resultados foram interpretados serialmente para priorizar com maior especificidade os sinais mais intensos. A base de dados utilizada tinha notificações coletadas pelo setor de Serviço de atendimento ao cliente e Farmacovigilância (SAC/FMV) de uma empresa farmacêutica veterinária no Brasil.

\section{MATERIAIS E MÉTODOS}

Foram usados os dados do SAC/FMV de uma empresa farmacêutica veterinária coletados entre 2014 e 2016 mediante questionários estruturados aplicados telefonicamente por entrevistadores. Médicos veterinários e assistentes foram devidamente treinados como entrevistadores para adquirir informações dos notificantes, seguindo como base o relatório de notificação de casos de farmacovigilância desenvolvido pela empresa. Também foram utilizados documentos complementares fornecidos pelos notificantes como os resultados laboratoriais, laudos post-mortem, fotos do produto, comprovantes de vacinação, receitas veterinárias ou notas fiscais. O corpo técnico veterinário da empresa avaliou o conjunto de dados para classificar os EA seguindo o fluxograma da Figura 1. As definições utilizadas estão descritas no Glossário. 


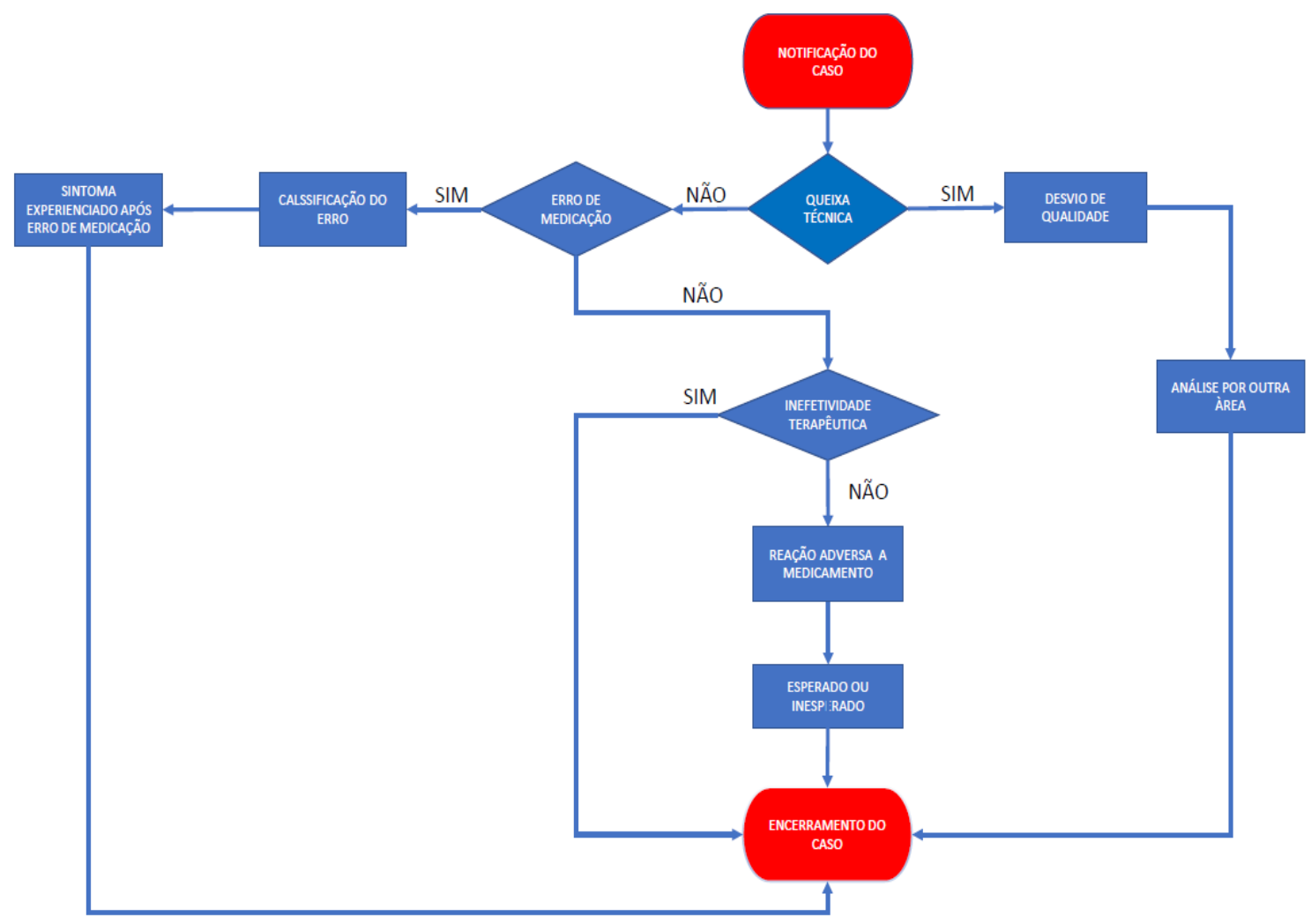

Figura 1 - Fluxograma para classificar eventos adversos

A avaliação de um caso utilizando o fluxograma serve para separar o tipo de notificação acolhida pela empresa, podendo ser utilizada durante o atendimento ou posteriormente. Isso auxilia em casos onde as notificações de EA já ocorreram em um banco de dados pré-definido. As classificações do fluxograma são compostas pelas caracterizações de EA determinados pela equipe técnica de farmacovigilância da empresa. A finalidade da figura 1 é classificar corretamente o caso buscando informações críticas dentro de uma anamnese e outros documentos de análise, de forma a diminuir análises de detecção de sinais errôneas, que pode culminar em ações de mitigação equivocadas. Os casos envolvendo vários animais de uma mesma propriedade que utilizaram um produto de partida similar foram tratados de forma individual. Não foram inseridos no estudo casos onde houve a confirmação de que o produto utilizado apresentava queixa técnica.

Após análise exploratória realizou-se a detecção de sinais por meio de 3 modelos: Reporting odds ratio (ROR), Bayesian confidence propagation neural network (BCPNN), e Gamma Poisson Shrinker (GPS). Os sinais detectados em cada método foram classificados de acordo com sua intensidade, sempre com o sinal mais intenso na primeira posição. Entre os sinais detectados pelos diferentes 
métodos, as posições de cada sinal foram somadas para obter uma classificação agregada que levasse em consideração os resultados dos três métodos.

$R O R$

O ROR é uma ferramenta de busca de sinais de farmacovigilância utilizada pelo EMA (CHAKRABORTY, 2015). O cálculo do ROR é análogo ao cálculo do Odds Ratio (OR) em estudos caso-controle, a partir de uma tabela de contingência (ROTHMAN, LANES E SACKS, 2004). A classificação dos sinais foi baseada no limite inferior do intervalo de confiança do ROR entre os sinais em que esse limite foi superior a 1,5 (primeiro lugar para o limite inferior de maior valor).

$B C P N N$

O BCPNN, empregado pela Organização Mundial de Saúde (OMS) (CHAKRABORTY, 2015), utiliza o logaritmo da razão entre a proporção observada de um binômio produto-EA específico, e a proporção esperada caso não houvesse associação entre o produto e o EA (KAJUNGU, SPEYBROECK, 2015). Valores positivos do logaritmo da razão anteriormente descrita ocorrem quando um binômio produto-EA tem frequência maior que o esperado em relação aos relatos gerais dos produtos e EA (KAJUNGU, SPEYBROECK, 2015). A classificação dos sinais utilizando o método BCPNN baseou-se na probabilidade posterior da hipótese nula, considerando apenas as combinações produto-EA com probabilidade inferior a $50 \%$ (primeiro lugar para a menor probabilidade).

GPS

O método GPS, utilizado pelo USFDA (CANIDA, 2017), é um método Bayesiano empírico que calcula o logaritmo do número observado do binômio produto-EA sobre o número de EA esperados com a finalidade de encontrar uma célula com um grande número de contagens em uma tabela de frequências de produtos e EA (KAJUNGU, SPEYBROECK, 2015). A classificação dos sinais utilizando o GPS também se baseou na probabilidade posterior da hipótese nula, considerando apenas as combinações produto-EA com probabilidade inferior a $50 \%$ (primeiro lugar para a menor probabilidade). 
Todas as análises foram realizadas com o Software R Core team (2017), utilizando os pacotes tidyverse 1.2.1 (WICKHAM, 2017) e PhViD 1.0.8 (AHMED, PONCET, 2016).

\section{RESULTADOS}

Dos 531 EA notificados, a ineficácia da vacina múltipla para cães foi o mais comum $(20,5 \%)$, seguido pela ineficácia da vacina múltipla para aves $(17,7 \%)$ e edema local de vacina múltipla para bovinos (7,9\%). Outros produtos-EA exibiram frequência inferior a 5\% (Tabela 1). As RAM representaram $217(40,8 \%)$ dos EA e foram classificadas em 18 categorias (Tabela 2), sendo que 81 (37\%) foram inesperadas. A maioria das RAM inesperadas (RAMI) se deu pelo vómito após administração de suplemento alimentar para cães e gatos (Tabela 3). Os erros de medicação representaram $8 \%$ (45) dos relatos e foram classificados em 5 categorias, sendo a administração em espécie não indicada a mais comum (Tabela 4).

Tabela 1 - Frequência dos relatos do binômio produto-EA. São Paulo, 2019.

\begin{tabular}{|c|c|c|c|}
\hline Produto & Eventos Adverso & Contagem & Frequência (\%) \\
\hline Vacina múltipla para cães & Ineficácia & 109 & 20,5 \\
\hline Vacina múltipla para aves & Ineficácia & 94 & 17,7 \\
\hline Vacina múltipla para bovinos & Edema local & 42 & 7,9 \\
\hline $\begin{array}{l}\text { Suplemento alimentar para cães e } \\
\text { gatos }\end{array}$ & Vómito & 24 & 4,5 \\
\hline Vacina para bovinos I & Edema local & 23 & 4,3 \\
\hline Vacina para aves I & Ineficácia & 18 & 3,4 \\
\hline $\begin{array}{l}\text { Associação de antiparasitários para } \\
\text { cães e gatos II }\end{array}$ & Intoxicação & 17 & 3,2 \\
\hline Antimicrobiano para cães e gatos I & Vómito & 16 & 3,0 \\
\hline Antiparasitário para cães & Intoxicação & 15 & 2,8 \\
\hline Vacina para bovinos I & Abscesso & 14 & 2,6 \\
\hline Vacina para aves II & Ineficácia & 13 & 2,4 \\
\hline Terapia hormonal para bovinos & Ineficácia & 12 & 2,3 \\
\hline $\begin{array}{l}\text { Associação de antiparasitários para } \\
\text { cães e gatos II }\end{array}$ & Vómito & 12 & 2,3 \\
\hline $\begin{array}{l}\text { Suplemento alimentar para cães e } \\
\text { gatos }\end{array}$ & Vômito e Diarreia associados & 11 & 2,1 \\
\hline Vacina para bovinos II & Ataxia & 10 & 1,9 \\
\hline Terapia hormonal para cães e gatos & Ineficácia & 9 & 1,7 \\
\hline $\begin{array}{l}\text { Associação de antiparasitários para } \\
\text { bovinos II }\end{array}$ & Intoxicação & 8 & 1,5 \\
\hline Vacina múltipla para cães & Anafilaxia & 8 & 1,5 \\
\hline
\end{tabular}


Associação de antiparasitários para cães e gatos I

Antiparasitário para bovinos I

Vacina para aves I

Vacina múltipla para bovinos

Vacina para bovinos I

Vacina múltipla para cães

Antimicrobiano para bovinos

Suplemento alimentar para cães e gatos

Vacina para bovinos I

Antiparasitário para bovinos II

Antiparasitário para bovinos II

Associação de antiparasitários para cães e gatos I

Associação de antiparasitários para cães III

Associação de antiparasitários para cães III

Antiparasitário para bovinos II

Antiparasitário para bovinos II

Associação de antiparasitários para bovinos II

Anti-inflamatório para cães

Antiparasitário para cães

Antiparasitário para bovinos III

Antiemético para cães e gatos

Antimicrobiano para cães II

Antimicrobiano para cães II

Associação de antiparasitários para cães e gatos I

Associação de antiparasitários para cães e gatos I

Associação de antiparasitários para cães e gatos I

Associação de antiparasitários para cães e gatos II

Associação de antiparasitários para cães e gatos II

Associação de antiparasitários para cães e gatos II

Associação de antiparasitários para cães e gatos II

Antimicrobiano para bovinos

Vacina para aves I

Vacina para bovinos II

Vacina múltipla para cães

Vacina para bovinos II

Vacina para bovinos II
Intoxicação

Intoxicação 6

Ataxia

Ataxia 6

1,1

Ineficácia

1,1

Sensibilidade Abdominal

0,9

Pirexia

Sensibilidade Local 3

Intoxicação

Reação Vacinal

0,6

Intoxicação

0,4

Vómito

0,4

Ineficácia

0,4

Ineficácia

Intoxicação

Hipersensibilidade

Ineficácia

0,2

Fadiga

0,2

Visão Subnormal

0,2

Prurido

Tremor

0,2

Hipersensibilidade

0,2

Ineficácia

0,2

Prurido

0,2

Diarreia

Hematoquezia

Vómito

Fadiga

Hematoquezia

Ineficácia

Prurido

0,2

Edema local

0,2

Morte súbita

0,2

Hematoma

0,2

Sensibilidade local

0,2

Anafilaxia

0,2

Ineficácia 
Tabela 2- Frequência dos relatos do binômio produto-RAM. São Paulo, 2019.

\begin{tabular}{|c|c|c|c|}
\hline Produto & RAM & Contagem & Frequência (\%) \\
\hline Vacina múltipla para bovinos & Edema local & 42 & 19,4 \\
\hline Vacina para bovinos I & Edema local & 23 & 10,6 \\
\hline Suplemento Alimentar para cães e gatos & Vómito & 19 & 8,8 \\
\hline Associação de antiparasitários para cães e gatos II & Intoxicação & 16 & 7,4 \\
\hline Antimicrobiano para cães e gatos I & Vómito & 16 & 7,4 \\
\hline Vacina para bovinos I & Abscesso & 14 & 6,5 \\
\hline Associação de antiparasitários para cães e gatos II & $\begin{array}{l}\text { Vómito } \\
\text { Vômito e } \\
\text { Diarreia } \\
\text { associados }\end{array}$ & 11 & 5,1 \\
\hline Vacina para bovinos II & Ataxia & 10 & 4,6 \\
\hline Vacina múltipla para cães & Anafilaxia & 8 & 3,7 \\
\hline Antiparasitário para cães & Intoxicação & 7 & 3,2 \\
\hline $\begin{array}{l}\text { Associação de antiparasitários para bovinos II } \\
\text { Vacina para bovinos I }\end{array}$ & $\begin{array}{l}\text { Intoxicação } \\
\text { Sensibilidade } \\
\text { Abdominal }\end{array}$ & $\begin{array}{l}6 \\
5\end{array}$ & 2,8 \\
\hline Vacina múltipla para cães & Pirexia & 5 & 2,3 \\
\hline Vacina para bovinos I & $\begin{array}{l}\text { Pirexia } \\
\text { Sensibilidade }\end{array}$ & 3 & 1,4 \\
\hline Antimicrobiano para bovinos & local & 3 & 1,4 \\
\hline Suplemento alimentar para cães e gatos & $\begin{array}{l}\text { Intoxicação } \\
\text { Sensibilidade }\end{array}$ & 3 & 1,4 \\
\hline $\begin{array}{l}\text { Vacina múltipla para cães } \\
\text { Associação de antiparasitários para cães III Antiparasitário } \\
\text { para bovinos III }\end{array}$ & $\begin{array}{l}\text { local } \\
\text { Intoxicação }\end{array}$ & $\begin{array}{l}1 \\
1\end{array}$ & $\begin{array}{l}0,5 \\
0,5\end{array}$ \\
\hline Anti-inflamatório para cães & Visão subnormal & 1 & 0,5 \\
\hline Vacina para bovinos II & Hematoma & 1 & 0,5 \\
\hline Associação de antiparasitários para cães e gatos I & Diarreia & 1 & 0,5 \\
\hline Associação de antiparasitários para bovinos II & Fadiga & 1 & 0,5 \\
\hline Associação de antiparasitários para cães e gatos II & Fadiga & 1 & 0,5 \\
\hline Antiparasitário para bovinos III & $\begin{array}{l}\text { Tremor } \\
\text { Hipersensibilida }\end{array}$ & 1 & 0,5 \\
\hline Antiemético para cães e gatos & de & 1 & 0,5 \\
\hline Associação de antiparasitários para cães e gatos I & Hematoquezia & 1 & 0,5 \\
\hline Associação de antiparasitários para cães e gatos II & Hematoquezia & 1 & 0,5 \\
\hline Associação de antiparasitários para cães e gatos II & Prurido & 1 & 0,5 \\
\hline $\begin{array}{l}\text { Vacina para bovinos II } \\
\text { Associação de antiparasitários para cães III Antiparasitário } \\
\text { para bovinos III }\end{array}$ & $\begin{array}{l}\text { Anafilaxia } \\
\text { Intoxicação }\end{array}$ & $\begin{array}{l}1 \\
1\end{array}$ & $\begin{array}{l}0,5 \\
0,5\end{array}$ \\
\hline Associação de antiparasitários para cães e gatos I & Intoxicação & 1 & 0,5 \\
\hline
\end{tabular}


Tabela 3 - Frequência dos relatos do binômio produto-RAMI. São Paulo, 2019.

\begin{tabular}{llcc} 
Princípio ativo & RAMI & Contagem & Frequência (\%) \\
\hline $\begin{array}{l}\text { Suplemento alimentar para cães e gatos } \\
\text { Associação de antiparasitários para cães e }\end{array}$ & Vómito & 24 & 30 \\
gatos II & Intoxicação & 12 & 15 \\
Associação de antiparasitários para cães e & Vómito & 12 & 15 \\
gatos II & Vômito e Diarreia & 11 & 14 \\
Suplemento alimentar para cães e gatos & associados & 6 & 7 \\
Antiparasitário para cães & Intoxicação & 6 \\
Vacina para bovinos I & Sensibilidade & 5 & 6 \\
Associação de antiparasitários para cães e & Abdominal & 5 & 4 \\
gatos II & Intoxicação & 3 & 2 \\
Vacina para bovinos I & Pirexia & 2 & 1 \\
Associação de antiparasitários para cães III & Intoxicação & 2 & 100 \\
Antiparasitário para bovinos III & Visão subnormal & 1 &
\end{tabular}

Tabela 4 - Frequência dos erros de medicação notificados. São Paulo, 2019.

\begin{tabular}{|c|c|c|c|c|}
\hline Produto & Erro de medicação & EA & Contagem & Frequência (\%) \\
\hline Antiparasitário para cães & $\begin{array}{l}\text { técnica de administração } \\
\text { errada }\end{array}$ & intoxicação & 8 & 18 \\
\hline $\begin{array}{l}\text { Associação de antiparasitários } \\
\text { para cães e gatos I }\end{array}$ & dosagem errada & intoxicação & 6 & 13 \\
\hline Vacina para aves I & prescrição inadequada & ataxia & 6 & 13 \\
\hline Antiparasitário para bovinos I & prescrição inadequada & intoxicação & 6 & 13 \\
\hline $\begin{array}{l}\text { Suplemento alimentar para } \\
\text { cães e gatos }\end{array}$ & dosagem errada & vómito & 5 & 11 \\
\hline $\begin{array}{l}\text { Associação de antiparasitários } \\
\text { para bovinos II }\end{array}$ & espécie não indicada & intoxicação & 2 & 4 \\
\hline Antiparasitário para bovinos II & espécie não indicada & vómito & 2 & 4 \\
\hline Antiparasitário para bovinos II & espécie não indicada & intoxicação & 2 & 4 \\
\hline $\begin{array}{l}\text { Associação de antiparasitários } \\
\text { para cães e gatos II }\end{array}$ & dosagem errada & intoxicação & 1 & 2 \\
\hline $\begin{array}{l}\text { Associação de antiparasitários } \\
\text { para cães e gatos II }\end{array}$ & dosagem errada & vómito & 1 & 2 \\
\hline Antimicrobiano para cães II & dosagem errada & prurido & 1 & 2 \\
\hline $\begin{array}{l}\text { Associação de antiparasitários } \\
\text { para cães e gatos I }\end{array}$ & dosagem errada & vómito & 1 & 2 \\
\hline Antiparasitário para bovinos II & espécie não indicada & $\begin{array}{l}\text { hipersensibili } \\
\text { dade }\end{array}$ & 1 & 2 \\
\hline Antimicrobiano para bovinos & prescrição inadequada & edema local & 1 & 2 \\
\hline Vacina para aves I & prescrição inadequada & morte súbita & 1 & 2 \\
\hline Antiparasitário para cães & $\begin{array}{l}\text { técnica de administração } \\
\text { errada }\end{array}$ & prurido & 1 & 2 \\
\hline Total & & & 45 & 100 \\
\hline
\end{tabular}

Dos 531 EA notificados, sete sinais foram identificados pela agregação na classificação dos três métodos de detecção de sinais. Desses 7 , quatro foram 
vacinas, um suplemento alimentar para cães e gatos, um vermífugo e um medicamento (Tabela 5).

Tabela 5 - Classificação dos sinais detectados de acordo com a força do sinal. São Paulo, 2019.

\begin{tabular}{llcccc}
\hline & & \multicolumn{3}{c}{ Classificação } \\
Produto & EA & ROR $^{*}$ & BCPNN $^{* *}$ & GPS $^{* * *}$ & Agregada \\
\hline Vacina múltipla para bovinos & Edema local & 1 & 1 & 6 & 8 \\
Suplemento alimentar para cães e & Vómito & 2 & 2 & 7 & 11 \\
gatos & Ataxia & 5 & 9 & 5 & 19 \\
Vacina para bovinos II & Edema local & 4 & 6 & 13 & 23 \\
Vacina para bovinos I & Ineficácia & 3 & 8 & 14 & 25 \\
Vacina múltipla para cães & Intoxicação & 7 & 7 & 11 & 25 \\
Antiparasitário para cães & Intoxicação & 6 & 11 & 15 & 32 \\
$\begin{array}{l}\text { Associação de antiparasitários para } \\
\text { cães e gatos II }\end{array}$ & & & & & \\
\end{tabular}

${ }^{*}$ Reporting odds ratio, ${ }^{* *}$ Bayesian Confidence Neural Network, ${ }^{* *}$ Gamma Poisson Shrinker

\section{DISCUSSÃO}

A análise dos dados do SAC/FMV de uma empresa farmacêutica veterinária no Brasil permitiu detectar sinais que poderão orientar futuras ações de farmacovigilância no escopo da empresa. Adicionalmente, os procedimentos utilizados para classificar os EA e para detectar e classificar os sinais poderão ser usados como referência na implementação de um sistema de farmacovigilância veterinária nacional.

Em relação às classificações das RAM, não se realizaram análises de causalidade. Apesar da definição de RAM ser baseada na existência de uma relação causal entre o medicamento e a suspeita, sabe-se que, se um evento for relatado espontaneamente pelo profissional de saúde ou pelo consumidor como fonte primária, mesmo que sua relação causal seja desconhecida ou não constatada, ela atende à definição de reação adversa (EMA,2017). O mesmo ocorre na tabela 3 , onde são apontadas as reações adversas inesperadas, eventos relatados espontaneamente apresentando uma reação diferente do previsto nos materiais informativos e estudos clínicos controlados. Apesar de ser uma caracterização primária, sem análises de causalidade (NARANJO et al,1981, KRAMMER et al, 1979), a identificação auxilia o serviço de farmacovigilância a 
refinar seus dados para melhorar a detecção de sinais. No entanto, as análises de causalidade devem fazer parte da continuidade do presente trabalho.

Os EA registrados definem o conjunto de possíveis sinais a serem detectados. Portanto, variações na forma de classificar os relatos em categorias de EA influenciam os resultados das análises de detecção de sinais. O fluxograma para classificar os relatos em categorias de EA no presente estudo foi elaborado para reduzir a variabilidade na classificação dos relatos. Os critérios usados no fluxograma foram baseados na literatura de farmacovigilância e permitiram explicitar uma sequência padronizada.

Apesar dos múltiplos relatos de EA, apenas sete foram classificados como sinais pelo procedimento adotado e o sinal mais forte não foi do EA notificado com maior frequência. A consideração das frequências observadas e esperadas concentra a atenção nas associações produto-EA no lugar da simples distribuição dos EA e facilita a análise sistemática de grandes volumes de dados. Porém, os métodos atuais de detecção, ao identificarem os sinais mais intensos, podem dificultar a detecção de outros sinais de que embora não sejam os mais intensos, são relevantes. Esse é um problema a ser abordado por futuras pesquisas metodológicas (WALLER et al, 2004).

As três métricas usadas para detectar sinais são amplamente aceitas e usadas como ferramentas de farmacovigilância, porém, não são as únicas (VAN PUIJENBROEK et al, 2002). As três métricas usadas, assim como o Proportional Reporting Ratio (PRR), a Q de Yule e o Qui-quadrado têm efetividade semelhante na detecção de sinais quando há pelo menos quatro casos por binômio produto-EA no banco de dados (WALLER et al, 2004). Como vários dos binômios analisados não atingiram essa frequência e não se tem identificado uma métrica que de forma consistente seja melhor que as outras, optou-se por usar mais de uma. Especificamente usaram-se três e os resultados foram interpretados em série (o sinal deve ser detectado por todos os métodos) a fim de aumentar a especificidade da detecção. A interpretação em série também diminui a sensibilidade, contudo, preferiu-se o aumento da especificidade não só para diminuir a probabilidade de falsos sinais, mas para reduzir o número de alvos de medidas de mitigação. Eventuais sinais não detectados (falsos negativos) e que persistirem após a aplicação das medidas de mitigação efetivas teriam maior probabilidade de serem detectados porque sua frequência esperada seria menor (e a razão 
observada/esperada maior) após a mitigação dos sinais detectados, isto é, a frequência esperada de um dado binômio produto-EA depende da frequência de outros binômios. Os binômios produto-EA mitigados passam a contribuir com frequências relativas menores em análises posteriores se as frequências dos binômios restantes permanecem inalteradas. Os sinais detectados foram classificados de acordo com a intensidade do sinal estimado por cada um dos três métodos e a soma dessas três intensidades determinou a classificação final de cada sinal. Essa classificação final pode ser vista com uma lista de sinais prioritários a serem mitigados.

$O$ aumento da especificidade teria sido maior se tivessem se interpretado em série os resultados de mais de três métodos de detecção de sinais. Nesse sentido, a escolha de apenas três métodos para aumentar a especificidade, sem prejudicar em tão alto grau a sensibilidade, foi uma escolha arbitrária. Também foi arbitrária a escolha do ROR, o BCPNN e o GPS, ao invés de outras métricas. No entanto, o principal propósito foi usar mais de um método para sugerir a interpretação em série e ter um ponto de partida para discutir estratégias de escolha baseada na especificidade, sensibilidade e valores preditivos da detecção.

A detecção de sinais foi baseada em relatos espontâneos, o qual melhorou o aproveitamento dos dados coletados de forma contínua. Os relatos espontâneos possibilitam uma farmacovigilância passiva que contribui a detecção de sinais não identificados na fase prévia à comercialização (BATE, EDWARDS, 2006). A facilidade de obtenção de relatos espontâneos se reflete no seu uso na literatura de estudos aplicados (BATE, EDWARDS, 2006). Por outro lado, a recente disponibilidade de softwares de acesso aberto com métodos de detecção de sinais facilita a análise de dados (BATE, EDWARDS, 2006). Se a isto se soma a descrição de procedimentos para classificar EA e integrar várias métricas de detecção de sinais, a farmacovigilância baseada em relatos espontâneos torna-se ainda mais acessível e informativa para as empresas farmacêuticas. Contudo, as limitações da vigilância passiva devem ser reconhecidas e complementadas com ações de busca ativa de sinais.

A detecção de sinais usou um único banco de dados com relatos espontâneos relativos a diferentes espécies. Análises estratificadas por espécie evitariam a influência das frequências observadas de binômios produto-EA de uma espécie nas frequências esperadas em outras espécies, levando à detecção de 
sinais espécie-específicos. A detecção de sinais também poderia ser complementada com a inclusão de covariáveis para melhorar seu desempenho (KAJUNGU, SPEYBROECK, 2015). Porém, o banco de dados utilizado tinha muitos binômios com frequências baixas que impossibilitaram a estratificação e uso de covariáveis.

Pelo conhecimento dos autores, o presente estudo é a primeira análise de farmacovigilância veterinária realizada no Brasil. É um estudo que serve como referência e estímulo para retomar a iniciativa da implementação de um sistema de farmacovigilância veterinária nacional. Um sistema de porte nacional permitiria a integração com sistemas de farmacovigilância humana (VARALLO et al, 2019), a padronização de procedimentos para analisar dados de diversas fontes e análises mais detalhadas mediante a estratificação e inclusão de covariáveis de bancos de dados maiores. A padronização de procedimentos deve incluir uma classificação de eventos adversos e a escolha de métodos de detecção de sinais pode ser guiada pela especificidade, sensibilidade e valores preditivos da detecção. 


\section{$5 \quad$ CONCLUSÃO}

Tendo em vista a importância da farmacovigilância na medicina veterinária e observando que não há uma diretriz da sua implementação e uso no Brasil, as técnicas abordadas neste trabalho podem apoiar o delineamento de um sistema de farmacovigilância para empresas do ramo veterinário e se tornar referência para a retomada da criação de um sistema de farmacovigilância nacional integrado. 
AHMED, I.; PONCET, A. R package phvid: an r package for pharmacovigilance signal detection. Version 1.0.8. 2016.

ANVISA - agência nacional de vigilância sanitária. Resolução $n^{\circ} \operatorname{rdc} n^{\circ} 4$, de 10 de fevereiro de 2009 - Guia regulatório - anvisa - glossário: normas de farmacovigilância para os detentores de registro de medicamentos de uso humano. Brasil: ministério da saúde, disponível em: <http://portal.anvisa.gov.br/documents/33868/2894051/gloss\%c3\%a1rio+da+resol u\%c3\%a7\%c3\%a3o+rdc+n\%c2\%ba+4\%2c+de+10+de+fevereiro+de+2009/61110 af5-1749-47b4-9d81-ea5c6c1f322a>. Acesso em: 25 ago. 2019.

ANVISA- agência nacional de vigilância sanitária. Rdc 17/2010: resolução rdc $\mathbf{n}^{\circ}$ 17, de 16 de abril de 2010. 2010. Disponível em: <http://portal.anvisa.gov.br/documents/33880/2568070/res0017_16_04_2010.pdf/b 9a8a293-f04c-45d1-ad4c-19e3e8bee9fa>. Acesso em: 19 ago. 2019.

BATE, A. AND EDWARDS, I. R., Data Mining in Spontaneous Reports. Basic \& Clinical Pharmacology \& Toxicology, 98: 324-330. doi:10.1111/j.17427843.2006.pto_232.x, 2006

BRASIL. Portaria n. 152, de 30 de nov. De 2008. Portaria sda/mapa 152/2008. Portaria em consulta pública. Brasília, p. 1-22, nov.2008.

CANIDA, T.; IHRIE, J. Openebgm: an r implementation of the gamma-poisson shrinker data mining model. The r journal, v. 9, n. 2, p. 499, 201. Disponível em: $<$ https://journal.r-project.org/archive/2017/rj-2017-063/rj-2017-063.pdf>.

CIOMS - council for international organizations of medical sciences. Practical aspects of signal detection in pharmacovigilance. Report of cioms working group viii, geneva, 2010.

CHAKRABORTY B, S. Pharmacovigilance: a data mining approach to signal detection. Indian j pharmacol, pág 47:241-2 2015

EMA - european medicines agency. Recommendation on pharmacovigilance surveillance and signal detection of veterinary medicinal products. Committee for Medicinal Products for Veterinary Use.13 de maio de 2015. 
EMA - european medicines agency. Inspections, human medicines, pharmacovigilance and committees division. Screening for adverse reactions in eudravigilance. London, uk. 2016

EMA - european medicines agency. Ema/876333/2011: guideline on good pharmacovigilance practices (gvp) annex $\mathbf{i}$ - definitions (rev 4). N/a, 2017. Disponível em: <https://www.ema.europa.eu/en/documents/scientificguideline/guideline-good-pharmacovigilance-practices-annex-i-definitions-rev4_en.pdf>. Acesso em: 19 ago. 2019.

FUSCO, M.A; OLIVEIRA, C.V.S; PEPE, V.L.E. farmacovigilância veterinária e a saúde humana: uma revisão dos programas selecionados de notificação de eventos adversos a medicamentos veterinários v.15, n.1, p.49-61, 2010.

KAJUNGU, D.K.; SPEYBROECK, N. Implementation of signal detection methods in pharmacovigilance - a case for their application to safety data from developing countries. In: journal of biometrics \& biostatistics, vol. 2, no.2, p. $5 p, 2015$

Kramer MS, Leventhal JM, Huchinson TA, et al. An algorithm for the operational assessment of adverse drug reactions. 242: 623-32

https://www.ncbi.nlm.nih.gov/pubmed/449002?dopt=Abstract. JAMA 1979

LIMA, P. F. D. et al. Queixas técnicas e eventos adversos a medicamentos notificados em um hospital sentinela do interior de são paulo, 2009-2010. Epidemiol. Serv. Saúde [online]. Vol.22, n.4, pp.679-686, 2013.

MINISTÉRIO DA SAÚDE. Fundação nacional de saúde centro nacional de epidemiologia. Guia de vigilância epidemiológica volume i. Aids / hepatites virais. Brasília: ministério da saúde, p -447, 2002.

MINISTÉRIO DA SAÚDE. Rdc 51/2014: resolução da diretoria colegiada - rdc $\mathbf{n}^{\circ}$ 51, de 29 de setembro de 2014. Brasília: diário oficial da união, 2014a. Disponível em:

<http://bvsms.saude.gov.br/bvs/saudelegis/anvisa/2014/rdc0051_29_09_2014.html >. Acesso em: 19 ago. 2019.

MINISTÉRIO DA SAÚDE. Secretaria de vigilância em saúde. Manual de eventos adversos pós-vacinação. Brasília: ministério da saúde, 2014b. 254 p. 
MOTA, D. M. Evolução e resultados do sistema de farmacovigilância do Brasil. Tese (Doutorado), 290, 2017

Naranjo CA, Busto $U$, Sellers EM et al. "A method for estimating the probability of adverse drug reactions". Clin. Pharmacol. Ther. 30 (2): 239-45. doi:10.1038/clpt.1981.154. 1981

PETERLINI, M. A. S.; PEREIRA, S. R. Erros de medicação. Acta paul. Enferm, v. 15, n. 2, p. 7-8, 2002.

R CORE TEAM. R: A language and environment for statistical computing. $R$ Foundation for Statistical Computing, Vienna, Austria. 2019.

ROTHMAN, K.J; LANES, S.; SACKS, S.T. the reporting odds ratio and its advantages over the proportional reporting ratio. Pharmacoepidemiology and drug safety, boston, usa, v. 13, p. 519-523, jan. 2004.

VAN PUIJENBROEK, E. P., BATE, A., LEUFKENS, H. G., LINDQUIST, M., ORRE, R. AND EGBERTS, A. C. A comparison of measures of disproportionality for signal detection in spontaneous reporting systems for adverse drug reactions. Pharmacoepidem. Drug safe., 11: 3-10. Doi:10.1002/pds.668, 2002

VARALLO, F. R., FORGERINI, M., HERDEIRO, M. T., \& DE CARVALHO MASTROIANNI, P. (2019). Harmonization of Pharmacovigilance Regulation in Brazil: Opportunities to Improve Risk Communication. Clinical therapeutics, 41(3), 598-603.

WALLER, P., VAN PUIJENBROEK, E. P., EGBERTS, A. C. G., \& EVANS, S. The reporting odds ratio versus the proportional reporting ratio:'deuce'. Pharmacoepidemiology and drug safety, 13(8), 525-526, 2004

WICKHAM, H. R package tidyverse: easily install and load the 'tidyverse'. Version 1.2.1. 2017. Disponível em: <https://cran.r-project.org/package=tidyverse>.

WHO - World health organization. Essential medicines and health products. Pharmacovigilance. Disponível em: <http://www.who.int/medicines/areas/quality_safety/safety_efficacy/pharmvigi/en/>. Acesso em: 30 abr. 2018 


\section{APÊNDICE}

\section{Glossário}

Desvio de Qualidade: Qualquer afastamento dos parâmetros de qualidade estabelecidos para um produto ou processo. Ex: Alteração de aspecto; cor, odor; presença de partículas; suspeita de contaminação. (ANVISA, 2010)

Erro de medicação: Qualquer erro na prescrição, dispensação ou administração de um medicamento enquanto sob controle do profissional de saúde, paciente ou consumidor. (PETERLINI, PEREIRA, 2002)

Evento Adverso: Qualquer sinal desfavorável (incluindo um sinal anormal no exame laboratorial, por exemplo), sintoma ou doença temporalmente associada à utilização de um medicamento, quer seja ou não considerados relacionados com o medicamento. (ANVISA, 2009)

Inefetividade terapêutica: Ausência ou redução da resposta terapêutica esperada de um medicamento, sob as condições de uso prescritas ou indicadas em bula. (MINISTÉRIO DA SAÚDE, 2002)

Queixa Técnica: Alteração ou irregularidade de um produto relacionada a aspectos técnicos ou legais e que poderá ou não causar danos à saúde individual e coletiva. (MINISTÉRIO DA SAÚDE, 2014a)

Reação adversa medicamentosa (RAM): Qualquer resposta a um fármaco que seja prejudicial e que ocorra nas doses normalmente utilizadas, caracterizado pelo fato de existir uma relação causal entre um medicamento e a suspeita de ocorrência (EMA, 2017)

Reação esperada: Reação de natureza ou gravidade conhecida conforme informações de segurança referenciada, por exemplo, em bula e materiais de embalagem diversos emitidos e aprovados pela empresa detentora do registro do produto. (EMA, 2017)

Reação inesperada: Reação imprevista, segundo os estudos que deram suporte à confecção e aprovação do produto. 
ANEXO

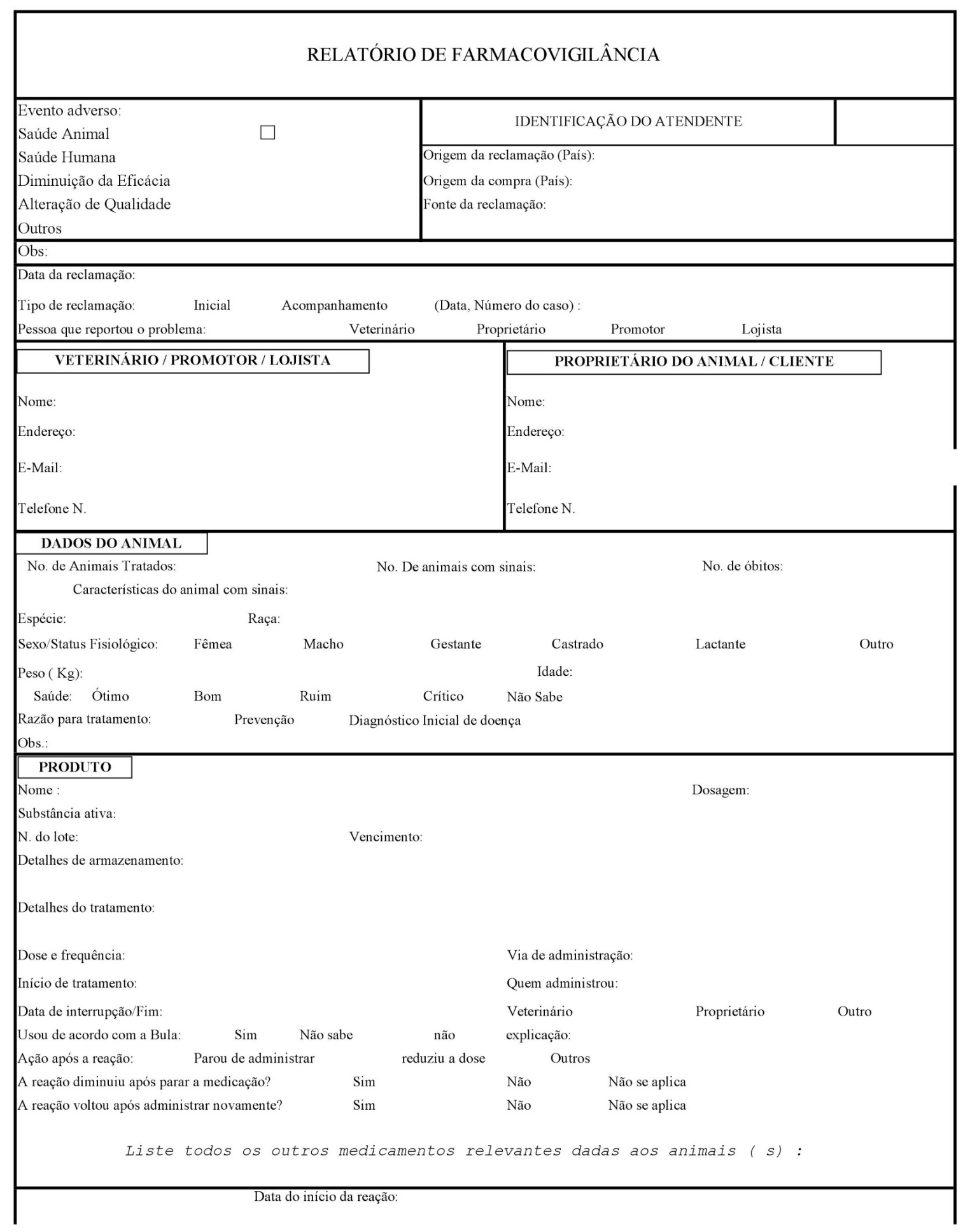


Duração da reação:

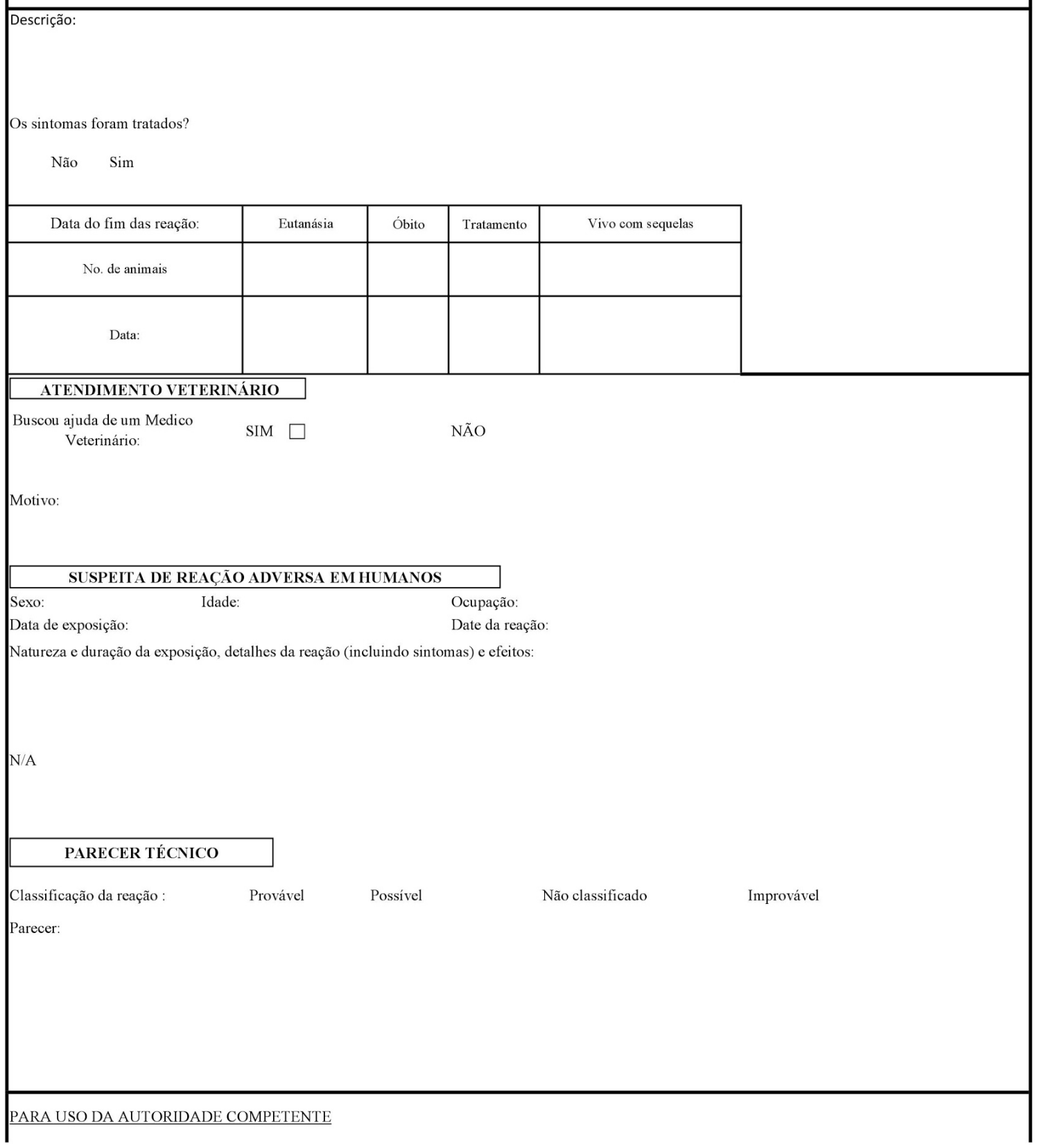


32

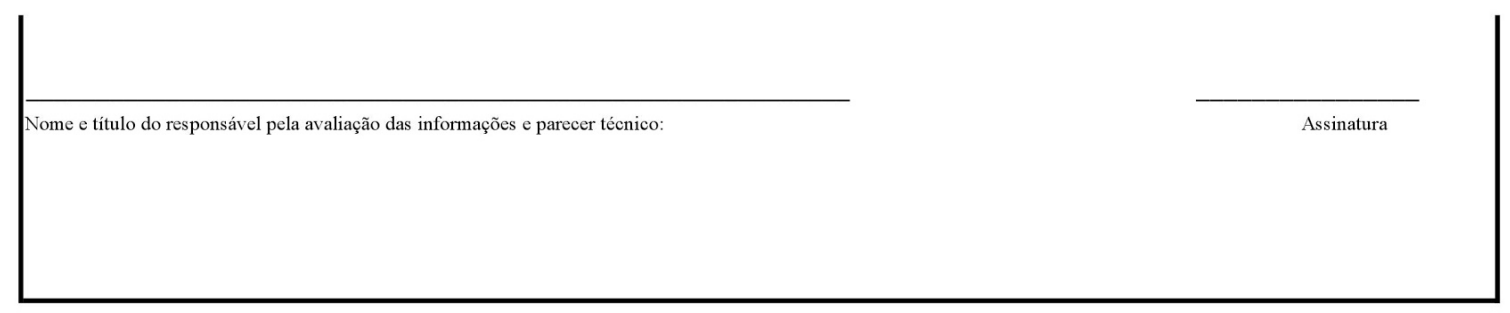

